\title{
Bambusae Caulis in Taeniam modulates neuroprotective and anti-neuroinflammatory effects in hippocampal and microglial cells via HO-1- and Nrf-2-mediated pathways
}

\author{
HYE WON EOM ${ }^{2 *}$, SUN YOUNG PARK ${ }^{1 *}$, YOUNG HUN KIM $^{1 *}$, SU JIN SEONG ${ }^{2}$, \\ MEI LING JIN ${ }^{2}$, EUN YEON RYU ${ }^{1}$, MIN JU KIM ${ }^{1}$ and SANG JOON LEE ${ }^{2}$ \\ ${ }^{1}$ Bio-IT Fusion Technology Research Institute and ${ }^{2}$ Department of Microbiology, \\ Pusan National University, Busan, Republic of Korea
}

Received June 12, 2012; Accepted August 10, 2012

DOI: $10.3892 /$ ijmm.2012.1128

\begin{abstract}
Recent evidence indicates that microglial activation and hippocampal damage may play important roles in neurodegenerative diseases, including Alzheimer's disease. Bambusae Caulis in Taeniam has been used as a folk remedy for the treatment of hypertension and cardiovascular disease in China and Korea. In this study, the mechanism responsible for the neuroprotective and anti-neuroinflammatory effects of Bambusae Caulis in Taeniam ethyl acetate fraction (BCE) was investigated. Heme oxygenase-1 (HO-1) is an inducible enzyme expressed in response to various inflammatory stimuli. Due to its role in the anti-inflammatory signaling pathway, the expression and modulation of HO-1 are important. In this study, the neuroprotective and antineuroinflammatory effects of BCE were examined using the murine microglial BV2 and hippocampal HT22 cells. We demonstrated that the administration of BCE provided neuroprotective effects against glutamate-induced cytotoxicity in HT22 cells through the HO-1 and nuclear erythroid-2 related factor 2 (Nrf-2) signaling pathways. We also reported that BCE inhibited lipopolysaccharide (LPS)-induced pro-inflammatory cytokines and that the presence of selective inhibitors of $\mathrm{HO}-1$ (SnPP) resulted in the inhibition of BCE-mediated anti-inflammatory activity in BV2 microglial cells. BCE was shown to induce HO-1 expression as well as the nuclear translocation of Nrf-2 in both microglial and hippocampal cells. These findings revealed the potential therapeutic mechanisms of BCE in neurodegenerative diseases, suggesting that HO-1
\end{abstract}

Correspondence to: Professor Sang Joon Lee, Department of Microbiology, Pusan National University, Busan 609-735, Republic of Korea

E-mail: sangjoon@pusan.ac.kr

*Contributed equally

Key words: microglial cells, hippocampal cells, heme oxygenase-1, nuclear erythroid-2 related factor 2, Bambusae Caulis in Taeniam and Nrf-2 signaling may play important roles in the mediation of its neuroprotective and anti-neuroinflammatory effects.

\section{Introduction}

Alzheimer's disease (AD) is the most common neurodegenerative disease causing adult dementia. The pathological feature of $\mathrm{AD}$ is progressive neuronal degeneration in separate areas of the forebrain, including the hippocampus and associated cortices (1). Oxidative stress has also been implicated in the pathophysiological mechanisms underlying AD (2). Thus, the regulation of oxidative stress is important in $\mathrm{AD}$ patients.

Microglia are the primary immune cells of the brain and play an essential role in the regulation of the immune response triggered by damaged cells (3). Microglial cells are considered 'brain macrophages' because they scavenge dying cells in the brain. However, chronic activation of these cells leads to the production of many pro-inflammatory cytokines and inflammatory mediators, such as nitric oxide (NO), tumor necrosis factor- $\alpha$ (TNF- $\alpha$ ), interleukin (IL)-1 $\beta$ and IL-6 (4). Therefore, the regulation of microglial activation has been regarded as an important therapeutic approach for the treatment of inflammatory neurodegenerative diseases. A number of studies have been conducted using BV2 cells, an immortalized murine microglial cell line. In addition to microglia, the hippocampus, an essential area for memory function, also has an important function pertinent in neurodegenerative diseases. Hippocampal HT22 cells are derived from the mouse hippocampus and have been used as a model demonstrating the mechanism of glutamate-induced neuronal cytotoxicity (5). Glutamate causes neuronal cell death within the nervous system. Moreover, it induces oxidative injury by suppressing the cellular uptake of cysteine through the cysteine/glutamate transport system, which in turn induces the depletion of glutathione and the accumulation of reactive oxygen species (ROS) (6). Thus, prevention of glutamate-induced oxidative damage in hippocampal cells may be an effective approach for arresting the progression of neurodegenerative disorders.

Nuclear erythroid-2 related factor 2 (Nrf-2) tightly regulates ROS levels (7). Nrf-2 is a redox-sensitive transcription factor modulating the cellular defense signaling pathway 
against toxic stimuli in several types of cells and tissues. This function of Nrf-2 has been demonstrated in several studies, including one which showed that Nrf-2 knockout mice were more vulnerable to oxidative damage (8). Under oxidative stress, the cytoplasmic Kelch-like ECH-associated protein 1 (Nrf-2/Keap1) perceives changes in the cellular environment, and its ability to promote Nrf-2 turnover is reduced; then, $\mathrm{Nrf}-2$ translocates into the nucleus and upregulates the expression of target genes containing the antioxidant response element (ARE) (9). Heme oxygenase-1 (HO-1) belongs to the family of ARE-containing genes and it is considered to be regulated by $\mathrm{Nrf}-2$. HO-1 is a stress-inducible protein that protects cells against inflammatory and oxidative stimuli. It catalyzes the oxidation of the heme molecule and decomposes the pro-oxidant heme into iron, carbon monoxide $(\mathrm{CO})$, biliverdin $(\mathrm{BV})$ and bilirubin $(\mathrm{BR})$ in the brain and in other tissues (10). The end-products of the HO-1 enzymatic process have anti-inflammatory activities which protect cells from oxidative stress. HO-1 has been implicated in aging-related neurodegenerative diseases including stroke, amyotrophic lateral sclerosis, Parkinson's disease (PD) and AD (11). Moreover, studies have demonstrated the neuroprotective aspects of HO-1 expression under different experimental conditions $(12,13)$.

Bambusae Caulis in Taeniam (BC) is the stem of Phyllostachys nigra var. henonis or Phyllostachys bambusoides (Family: Poaceae) and is a well-known traditional herbal medicine in the Orient (14). It has been used as a folk remedy for treating hypertension and cardiovascular disease (15). Oral doses of $5,000 \mathrm{mg} / \mathrm{kg}$ or less of BC did not produce toxic effects in rats, and the minimal lethal dose was discovered to be over $5,000 \mathrm{mg} / \mathrm{kg}$ body weight for both genders (16). However, a limited number of reports on the efficacy of the anti-inflammatory and neuroprotective functions of $\mathrm{BC}$ exist. Therefore, we examined whether Bambusae Caulis in Taeniam ethyl acetate fraction (BCE) induces HO-1 expression using the Nrf2 signaling pathway in order to regulate the neuroprotective and anti-neuroinflammatory effects in microglial BV2 and hippocampal HT22 cells.

\section{Materials and methods}

Cell culture. Microglial BV2 and hippocampal HT22 cell lines obtained from the American Type Culture Collection (Rockville, MD, USA) were grown as monolayers in Dulbecco's modified Eagle's medium (DMEM; Gibco-BRL, Carlsbad, CA, USA) supplemented with 10 or $5 \%$ heatinactivated fetal bovine serum (FBS; Gibco-BRL). The cells were incubated at $37^{\circ} \mathrm{C}$ in a humidified atmosphere containing $5 \% \mathrm{CO}_{2}$. To avoid changes in cell characteristics caused by extended periods of cell culture, all experiments were conducted with cells between passages 15 and 25 . Each cell suspension was subcultured by trypsin/EDTA treatment every 2 days in order to maintain exponential growth.

Cell viability assay. The cells were plated in 24-well plates at a density of $2 \times 10^{5}$ cells/well. 3-(4,5-Dimethylthiazol-2-yl)2,5-diphenyltetrazolium bromide (MTT; $50 \mu \mathrm{g} / \mathrm{ml}$ ) was added to each well. The plates were incubated for $4 \mathrm{~h}$ at $37^{\circ} \mathrm{C}$ in a $5 \% \mathrm{CO}_{2}$ atmosphere, after which the supernatant was removed and the formazan crystals that had formed in the viable cells were solubilized with dimethyl sulfoxide (DMSO). The absorbance of each well was then read at $570 \mathrm{~nm}$ using an enzyme-linked immunosorbent assay (ELISA) reader (Wallace, Boston, MA, USA).

Measurement of TNF- $\alpha, I L-1 \beta$ and IL- 6 concentrations. Cells were incubated first with various concentrations of $\mathrm{BCE}$ for $1 \mathrm{~h}$ and then with lipopolysaccharide (LPS) for $16 \mathrm{~h}$. Following the $24-\mathrm{h}$ incubation, TNF- $\alpha$, IL-1 $\beta$ and IL-6 levels in the culture media were quantified using an enzymelinked immunosorbent assay (ELISA) kit (R\&D Systems, Minneapolis, MN, USA) according to the manufacturer's instructions.

Measurement of ROS. To evaluate the levels of intracellular ROS, cells were treated with CM-H2DCFDA, an indicator of general oxidative stress, for $1 \mathrm{~h}$ at $37^{\circ} \mathrm{C}$ under $5 \% \mathrm{CO}_{2}$. The cells were then harvested and washed three times with phosphate-buffered saline (PBS). The fluorescence intensity was then measured by confocal microscopy, microplate fluorimetry and flow cytometry at an excitation wavelength of $488 \mathrm{~nm}$ and an emission wavelength of $525 \mathrm{~nm}$. Data analyses were performed using the CXP 2.0 software package (Beckman Coulter).

Transient transfection and dual luciferase assay. Cells were transfected with an ARE-reporter plasmid (Stratagene, Grand Island, NY, USA) using the FuGENE-HD reagent (Roche Applied Sciences) according to the manufacturer's instructions. Then, a Renilla luciferase control plasmid, pRL-CMV (Promega Corporation, Madison, WI, USA), was co-transfected as an internal control for verification of transfection efficiency. Twenty-four hours after transfection, the cells were incubated with BCE for $1 \mathrm{~h}$. Luciferase activity was assayed using a dual Luciferase assay kit (Promega Corporation) according to the manufacturer's instructions. Luminescence was measured using a microplate luminometer (Wallac 1420 multilabel counter; Perkin-Elmer, Downers Grove, IL, USA).

Western blot analysis. Cells were harvested in ice-cold lysis buffer consisting of $1 \%$ Triton X-100, $1 \%$ deoxycholate and $0.1 \%$ SDS. The protein content of the cell lysates was then determined using Bradford reagent (Bio-Rad, Hercules, CA, USA). Total proteins in each sample $(50 \mu \mathrm{g})$ were resolved by 7.5\% SDS-polyacrylamide gel electrophoresis (SDS-PAGE), transferred to a polyvinylidene difluoride (PVDF) membrane and incubated with the appropriate antibodies. The proteins were then visualized using an enhanced chemiluminescence detection system (Amersham Biosciences, Piscataway, NJ, USA) with horseradish peroxidase-conjugated anti-rabbit or anti-mouse secondary antibodies. Images were acquired using an ImageQuant 350 analyzer (Amersham Biosciences).

Quantitative real-time polymerase chain reaction ( $q R T-P C R$ ). Total RNA was isolated from the cells using the RNAspin Mini RNA Isolation kit (GE Healthcare, Buckinghamshire, UK) according to the manufacturer's instructions. The cDNA was synthesized from $1 \mu \mathrm{g}$ of the total RNA using the Maxime RT PreMix (Intron Biotechnology, Seongnam, Korea) and anchored oligo(dT) $)_{15}$-primers. Real-time PCR was performed 

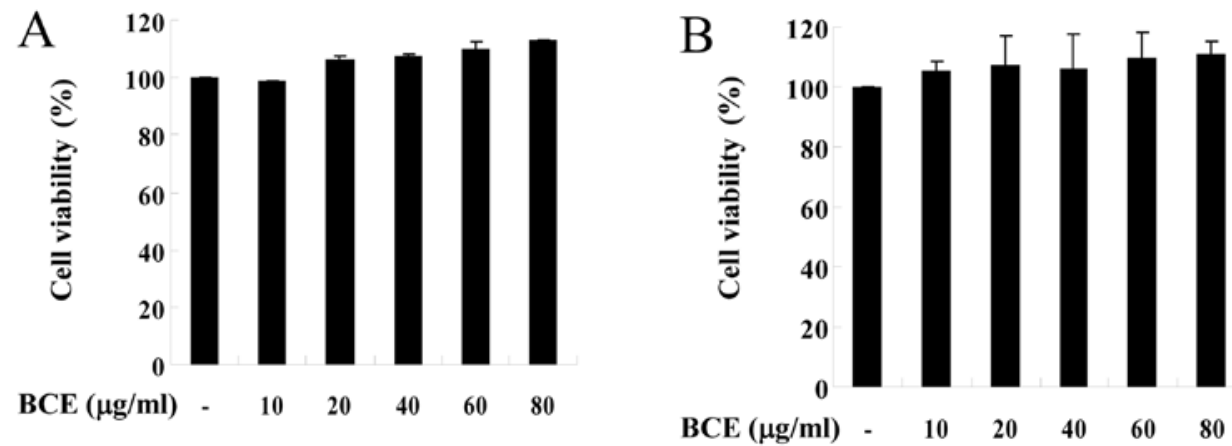

Figure 1. Effects of BCE on the viability of microglial BV2 and hippocampal HT22 cells. (A) Microglial BV2 and (B) hippocampal HT22 cells were incubated with $0,10,20,40,60$ and $80 \mu \mathrm{g} / \mathrm{ml}$ of BCE for $24 \mathrm{~h}$. The cell viability was assessed using an MTT-based assay and the results are expressed as the percentage of surviving BCE-treated cells, compared to the BCE-untreated control cells.

using a Chromo4 ${ }^{\mathrm{TM}}$ instrument (Bio-Rad) using the SYBRGreen master mix (Applied Biosystems, Foster City, CA, USA). The relative amount of target mRNA was determined using an established comparative threshold $(\mathrm{Ct})$ method by normalizing the target mRNA Ct values to those for glyceraldehyde 3-phosphate dehydrogenase (GAPDH) $(\Delta \mathrm{Ct})$.

Statistical analyses. The data are expressed as the means \pm standard error (SE). Each experiment was repeated at least three times. Statistical analysis was performed using SPSS version 16.0 software (SPSS, Inc., Chicago, IL, USA). Significant differences were evaluated using one- or two-way ANOVA followed by the Dunn's post hoc test. P-values $<0.05$ were considered to represent statistically significant differences.

\section{Results}

Effects of BCE on microglial BV2 and hippocampal HT22 cell viability. Microglial cells play an essential role in the central nervous system, and hippocampal cells are important to memory function $(17,18)$. Therefore, it is of note that BCE does not produce cytotoxicity in microglial BV2 and hippocampal HT22 cells. To examine the potential cytotoxic properties of BCE, we first measured the effect of BCE on cell viability in BV2 and HT22 cells using the MTT assay. Cells were treated with $10,20,40,60$ and $80 \mu \mathrm{g} / \mathrm{ml}$ of BCE for $24 \mathrm{~h}$, followed by incubation with a working solution of MTT for $4 \mathrm{~h}$ at $37^{\circ} \mathrm{C}$. BCE did not affect cell viability in either microglial BV2 or hippocampal HT22 cells (Fig. 1A and B). Thus, our data suggest that $\mathrm{BCE}$ is non-toxic in doses ranging from $10-80 \mu \mathrm{g} / \mathrm{ml}$. Based on these results, we selected $10-80 \mu \mathrm{g} / \mathrm{ml}$ of BCE for use in subsequent experiments.

BCE suppresses LPS-induced pro-inflammatory mediators and cytokines in microglial BV2 cells. Under inflammatory conditions, such as exposure to LPS, activated microglia produce pro-inflammatory mediators and cytokines, including NO, TNF- $\alpha$, IL-1 $\beta$ and IL-6 (19). Since overproduction of these pro-inflammatory mediators and cytokines causes acute damage in cells, their production must be tightly controlled. Therefore, we investigated whether BCE regulates the expression of these inflammatory mediators and cytokines using western blotting and ELISA assays. Microglial BV2 cells were treated with BCE (40, 60 and
$80 \mu \mathrm{g} / \mathrm{ml}$ ) for $1 \mathrm{~h}$ and/or LPS at a dose of $1 \mu \mathrm{g} / \mathrm{ml}$ for $16 \mathrm{~h}$. NO production increased $\sim$-fold only in LPS-stimulated cells (Fig. 2A). However, the cells pre-treated with BCE demonstrated lower NO production when compared to cells treated with LPS alone, in a dose-dependent manner. Production of LPS-induced pro-inflammatory cytokines, including TNF- $\alpha$ (Fig. 2C), IL-1 $\beta$ (Fig. 2D) and IL-6 (Fig. 2E), decreased in the BCE-treated cells in a concentration-dependent manner. Next, we monitored the levels of inducible NO synthase (iNOS) and cyclooxygenase (COX)-2 by western blotting. Microglial BV2 cells were pre-treated with $0,10,20,40,60$ and $80 \mu \mathrm{g} / \mathrm{ml}$ of $\mathrm{BCE}$ and then incubated for $24 \mathrm{~h}$ with LPS $(1 \mu \mathrm{g} / \mathrm{ml})$. Levels of iNOS and COX-2 protein decreased in the BCE-treated BV2 cells, compared to those exposed only to LPS (Fig. 2B). These results indicated that $\mathrm{BCE}$ inhibited the LPS-induced production of pro-inflammatory mediators in microglial BV2 cells.

BCE protects hippocampal HT22 cells against glutamateinduced cell toxicity. Glutamate exposure leads to neuronal cell death in the nervous system (6). Glutamate exposure increases the generation of ROS, which, due to its reactive properties, causes cell death in severe cases. Therefore, we examined the neuroprotective properties of BCE in glutamate-stimulated hippocampal HT22 cells. The hippocampal HT22 cells were pre-treated with BCE $(10-80 \mu \mathrm{g} / \mathrm{ml})$ for $1 \mathrm{~h}$ and then incubated with $5 \mathrm{mM}$ glutamate for $24 \mathrm{~h}$. As shown in Fig. 3A, only $50 \%$ of the cells treated with glutamate remained viable, compared to cells not treated with glutamate. However, treatment with $10-80 \mu \mathrm{g} / \mathrm{ml}$ of BCE improved the viability of glutamate-stimulated cells gradually, in a dose-dependent manner. Due to the unstable nature of ROS, their overproduction may cause neuronal cell toxicity (20). Therefore, we investigated whether glutamate-induced ROS production was reduced by BCE in hippocampal HT22 cells. We observed that ROS production increased in glutamatetreated hippocampal HT22 cells (Fig. 3B). However, cells treated with $10-80 \mu \mathrm{g} / \mathrm{ml}$ of BCE prior to glutamate exposure exhibited a gradual reduction in ROS production, down to the same level as cells that had not been stimulated with glutamate. Taken together, these results lead us to conclude that BCE has cytoprotective and ROS-attenuating effects in hippocampal HT22 cells. Thus, BCE is regarded as a potential therapeutic treatment against the oxidative effects of glutamate. 
A

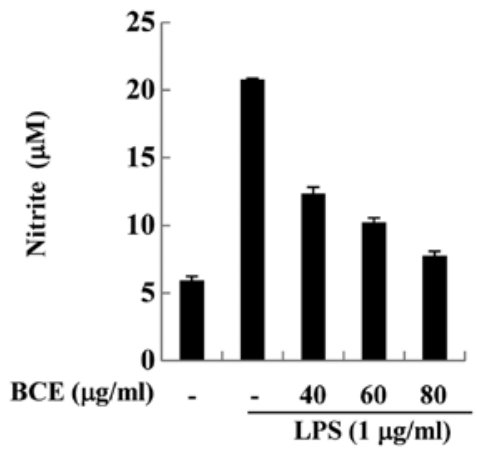

B

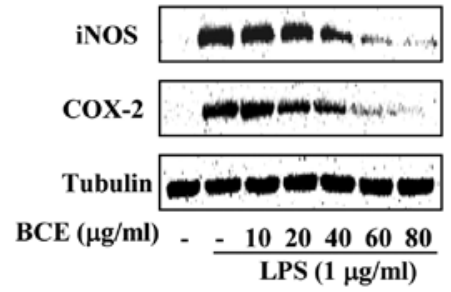

$\mathrm{C}$

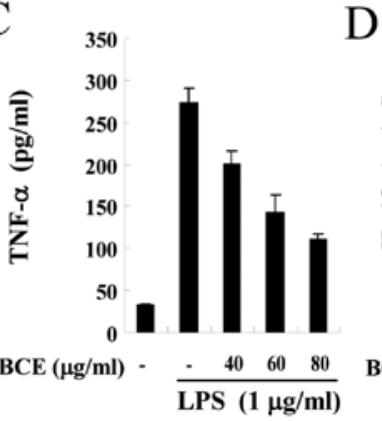

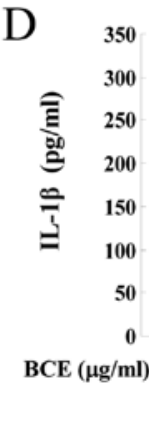
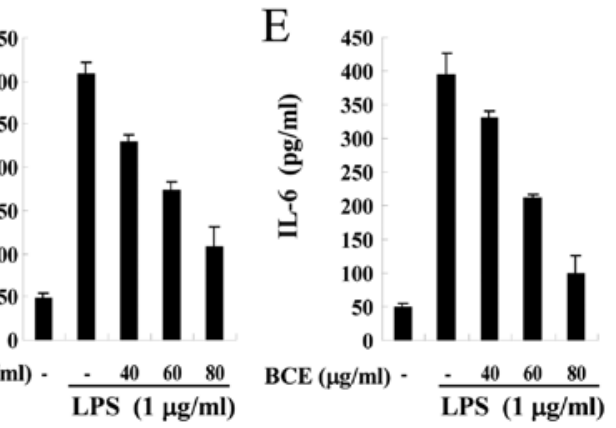

Figure 2. Effects of BCE on LPS-induced pro-inflammatory mediators in microglia BV2 cells. (A) BV2 cells were incubated with $0,40,60$ and $80 \mu \mathrm{g} / \mathrm{ml}$ of BCE for $1 \mathrm{~h}$, and then stimulated with LPS $(1 \mu \mathrm{g} / \mathrm{ml})$ for $16 \mathrm{~h}$. Nitrite production was measured using the Griess reaction. (B) BV2 cells were treated with various concentrations of BCE $(10,20,40,60$ and $80 \mu \mathrm{g} / \mathrm{ml})$ for $1 \mathrm{~h}$ prior to incubation with LPS $(1 \mu \mathrm{g} / \mathrm{ml})$ for $24 \mathrm{~h}$. Whole cell protein extracts were prepared and western blotting using antibodies specific for iNOS and COX-2 was conducted. Tubulin was used as a loading control. Cells were incubated with $0,40,60$ and $80 \mu \mathrm{g} / \mathrm{ml}$ of BCE for $1 \mathrm{~h}$ prior to treatment with LPS $(1 \mu \mathrm{g} / \mathrm{ml})$ for $24 \mathrm{~h}$. (C) TNF- $\alpha$, (D) IL-1 $\beta$ and (E) IL-6 were present in the culture supernatants. As measured by an ELISA assay.
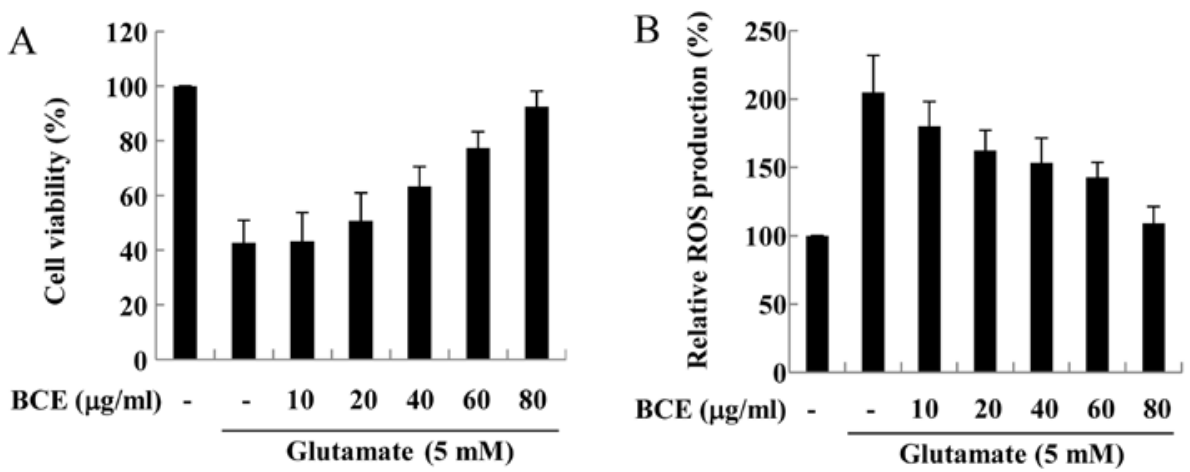

Figure 3. Effects of BCE on glutamate-induced oxidative neurotoxicity and ROS generation. HT22 cells were treated with BCE and then exposed to glutamate $(5 \mathrm{mM})$ for $24 \mathrm{~h}$. (A) Cell viability was measured by MTT assay as described in the Materials and methods. The results are expressed as the percentage of surviving cells compared to the control cells, which were not treated with BCE and glutamate. (B) HT22 cells were pre-treated with fluorescent dye and then either treated with BCE, or left untreated, prior to stimulation with glutamate $(5 \mathrm{mM})$. ROS production was determined by quantifying the resulting fluorescent dye using flow cytometry. The results are indicated as the percentage of fluorescence of treated cells over the fluorescence of the control cells, which were not treated with BCE and glutamate.

$B C E$ reduces $R O S$ production in microglial $B V 2$ and hippocampal HT22 cells. ROS is a by-product of oxygen metabolism in aerobic organisms and is considered an essential second messenger at low concentrations. However, higher concentrations of ROS have been reported to be harmful to cellular macromolecules (21). When there is an imbalance between the ROS generation and removal systems, neuronal cell damage occurs. Moreover, ROS are implicated in a number of neurodegenerative diseases including ischemia, PD and $\mathrm{AD}$, since ROS participate in chain reactions. Hence, we examined the effect of BCE on the elimination of ROS in LPS-stimulated microglial BV2 cells and glutamate- stimulated hippocampal HT22 cells using flow cytometry. ROS production was significantly increased in microglial BV2 cells stimulated by treatment with $1 \mu \mathrm{g} / \mathrm{ml}$ LPS for $12 \mathrm{~h}$, compared to cells not treated with LPS (Fig. 4A). However, ROS production was reduced in LPS-stimulated cells pretreated with BCE $(80 \mu \mathrm{g} / \mathrm{ml})$. The same experiment was conducted in hippocampal HT22 cells. ROS production in hippocampal HT22 cells exposed to $5 \mathrm{mM}$ glutamate for $12 \mathrm{~h}$ also increased, up to $\sim 3$ times that of cells not stimulated with glutamate, but pre-treatment of the hippocampal HT22 cells with $80 \mu \mathrm{g} / \mathrm{ml}$ of BCE decreased ROS production considerably (Fig. 4B). These results suggest that BCE exerts 

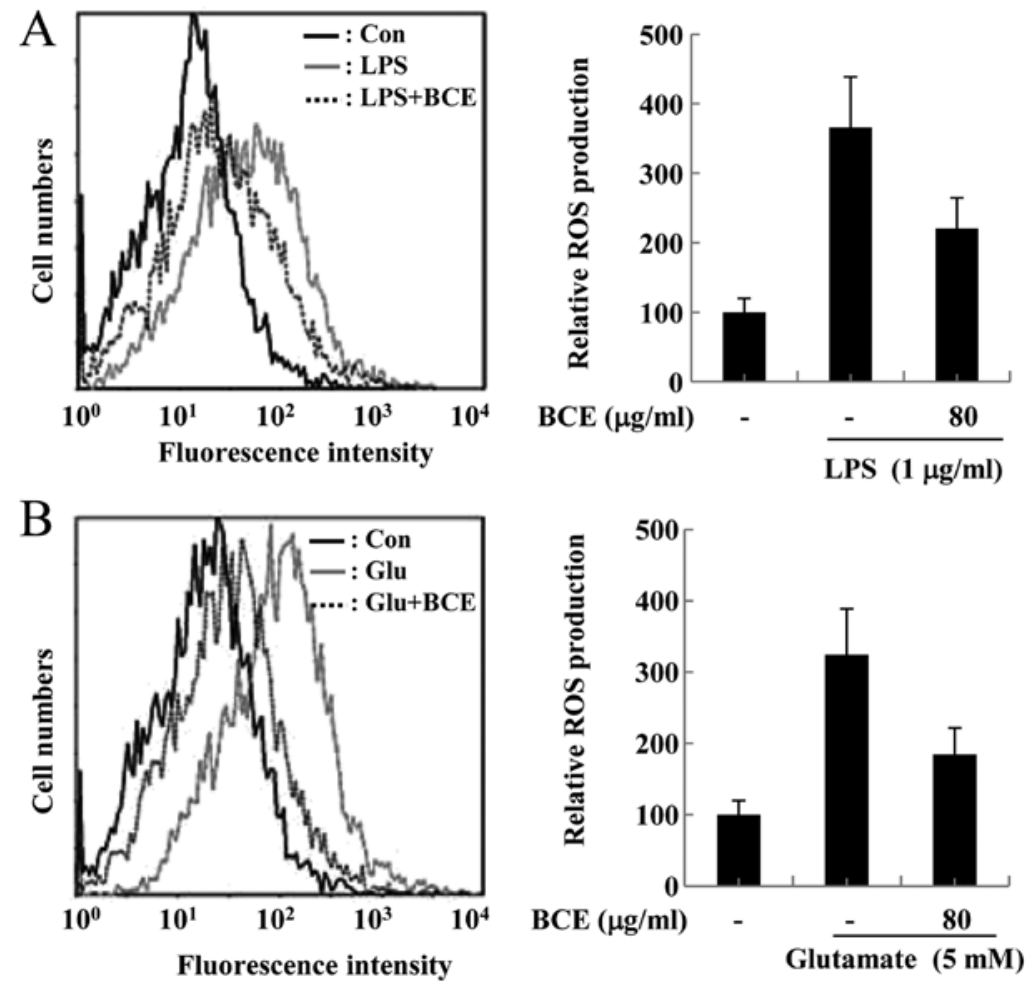

Figure 4. Effect of BCE on ROS production in microglial BV2 and hippocampal HT22 cells. Quantitative analysis of ROS production in both cells was conducted by flow cytometry. (A) Microglial BV2 and (B) hippocampal HT22 cells were pre-treated with $80 \mu \mathrm{g} / \mathrm{ml} \mathrm{BCE}$, or left untreated, prior to stimulation with LPS $(1 \mu \mathrm{g} / \mathrm{ml})$ or glutamate $(5 \mathrm{mM})$. Cells were then incubated with CM-H2DCFDA for an additional $1 \mathrm{~h}$. Intracellular levels of ROS were then determined by flow cytometry. The resulting fluorescence intensity represented the amount of ROS production in the cells. The results were indicated as the percentage of fluorescence intensity of the treated cells over the fluorescence of the control cells, which were not treated with BCE and LPS/glutamate.

neuroprotective and anti-neuroinflammatory effects through the restriction of ROS production.

$B C E$ treatment increases the $M R N A$ and protein expression levels of $\mathrm{HO}-1$ in microglial $\mathrm{BV} 2$ and hippocampal HT22 cells. HO-1 has been related to anti-inflammatory effects in recent studies $(11,12)$. HO-1 is also regarded as a cellular oxidative stress sensor since it responds to changes in the cellular redox status (13). Expression of HO-1 is restricted in normal brain neurons and neuroglia, but various inflammatory stimuli are known to induce HO-1 expression (22). An increase in HO-1 expression may protect cells by producing $\mathrm{BV}$ and BR, which are by-products of heme metabolism (10). To assess the effect of BCE on HO-1 protein expression, microglial BV2 and hippocampal HT22 cells were treated with $80 \mu \mathrm{g} / \mathrm{ml}$ of BCE for varying lengths of time $(0-24 \mathrm{~h})$. The HO-1 protein levels were increased by BCE treatment, especially in cells treated with BCE for $8 \mathrm{~h}$ (Fig. 5A and E). Next, we tested the protein expression level of HO-1 in both microglial BV2 and hippocampal HT22 cells treated with $0,10,20,40,60$ and $80 \mu \mathrm{g} / \mathrm{ml}$ of BCE and 0 and $20 \mu \mathrm{M}$ of CoPP (HO-1 activator) for $8 \mathrm{~h}$. HO-1 protein expression increased in the presence of $\mathrm{BCE}$, in a concentration-dependent manner (Fig. 5B and F). To confirm the effect of BCE on the expression of $\mathrm{HO}-1$ protein, we examined the $\mathrm{HO}-1$ mRNA level using qRT-PCR. The mRNA level of HO-1 increased in a dose-dependent manner in the presence of $\mathrm{BCE}$ (Fig. 5D and H). This data indicated that BCE induces HO-1 expression in microglial BV2 and hippocampal HT22 cells.
In other words, BCE may protect cells against inflammatory stimuli since it induces the expression of $\mathrm{HO}-1$, which has well-known anti-inflammatory and antioxidant effects.

Effects of BCE on the accumulation and transactivation function of Nrf-2 in microglial BV2 and hippocampal HT22 cells. Nrf-2 exists in an inactive form in the cytoplasm when bound by Keap1. However, when exposed to inflammatory stimuli, such as LPS and ROS, Nrf-2 is released from Keap1 and translocates from the cytoplasm into the nucleus (21). Nrf-2 plays a critical role in the induction of a number of genes associated with the antioxidant response, such as HO-1, since Nrf-2 is a transcription factor that binds to ARE in the promoter region of target genes (21). Nrf-2 is an important player in the upregulation of cellular stress-inducible genes, and the accumulation of Nrf-2 in the nucleus endows the cell with the adaptive response capability critical for maintaining normal cellular functions (23). We aimed to observe the effect of BCE on Nrf-2 nuclear accumulation and transactivation in microglial BV2 and hippocampal HT22 cells. First, microglial BV2 cells were treated with $80 \mu \mathrm{g} / \mathrm{ml}$ of BCE for $0.25-4 \mathrm{~h}$. $\mathrm{BCE}$ treatment increased nuclear accumulation of $\mathrm{Nrf}-2$ in these cells, especially in those treated with BCE for $0.5 \mathrm{~h}$ (Fig. 6A). When cells were treated with varying concentrations of $\operatorname{BCE}(10,20,40,60$ and $80 \mu \mathrm{g} / \mathrm{ml})$, while keeping the treatment time constant at $0.5 \mathrm{~h}, \mathrm{Nrf}-2$ nuclear accumulation was induced by BCE in a dose-dependent manner (Fig. 6B). Next, we performed the same experiments in hippocampal HT22 cells, treating them with $10-80 \mu \mathrm{g} / \mathrm{ml}$ of BCE for $4 \mathrm{~h}$. 
A

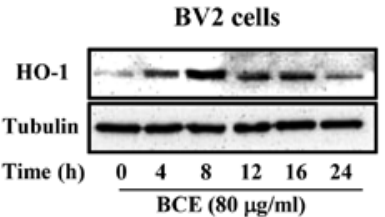

B

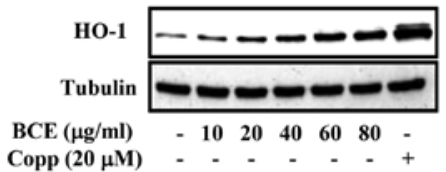

C

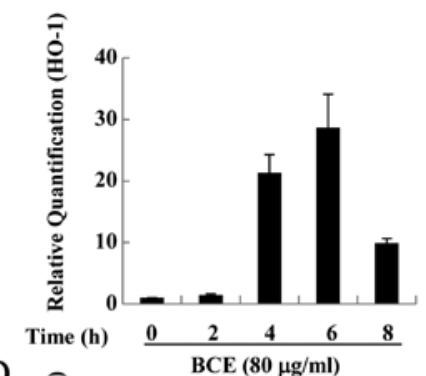

$\mathrm{D}$

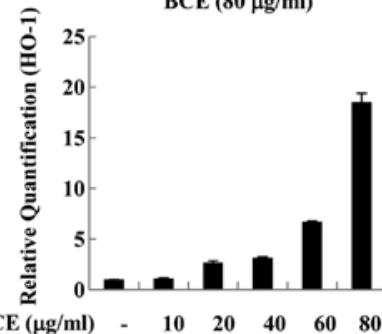

E

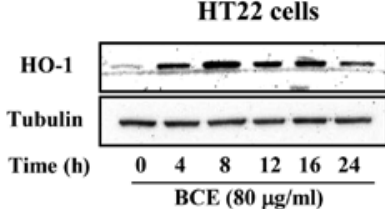

$\mathrm{F}$

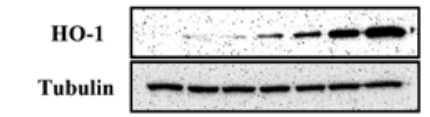

BCE $(\mu \mathrm{g} / \mathrm{ml}) \quad-102040 \quad 6080-$

Copp $(20 \mu \mathrm{M})$ - . . - . +
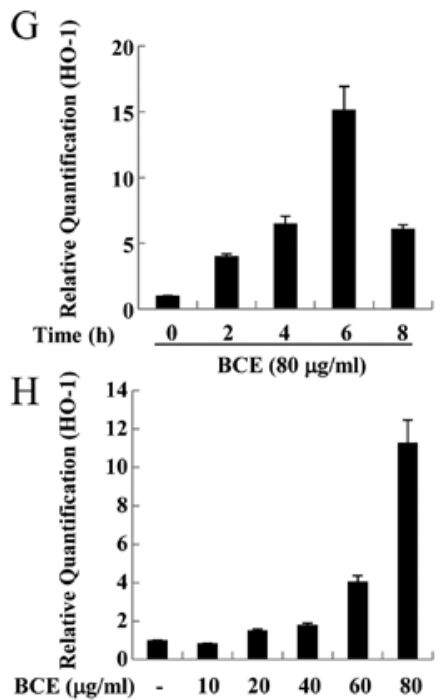

Figure 5. Treatment with BCE increased HO-1 mRNA levels and induced the expression of HO-1 in microglial BV2 and hippocampal HT22 cells. Whole cell protein extracts were analyzed by western blotting. (A and E) Microglial BV2 and hippocampal HT22 cells were treated with $80 \mu \mathrm{g} / \mathrm{ml}$ of BCE for 4-24 h. (B and F) Microglial BV2 and hippocampal HT22 cells were treated with BCE $(0,10,20,40,60$ and $80 \mu \mathrm{g} / \mathrm{ml})$ or CoPP (HO-1 activator) at 0 or $20 \mu \mathrm{M}$ for $8 \mathrm{~h}$. (C, D, G and H) HO-1 mRNA levels were measured by real-time qRT-PCR. Both cell types were treated with $80 \mu \mathrm{g} / \mathrm{ml}$ of BCE for $2-8 \mathrm{~h}$ or treated with $10,20,40,60$ and $80 \mu \mathrm{g} / \mathrm{ml}$ of BCE for $6 \mathrm{~h}$.

A

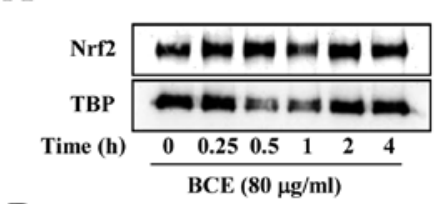

B

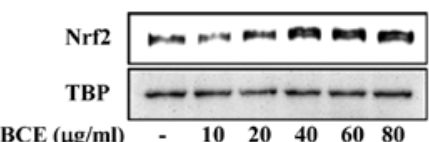

$\mathrm{E}$

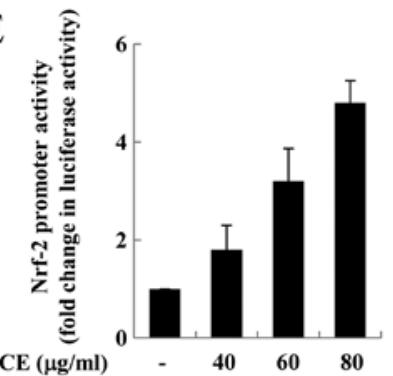

$\mathrm{C}$

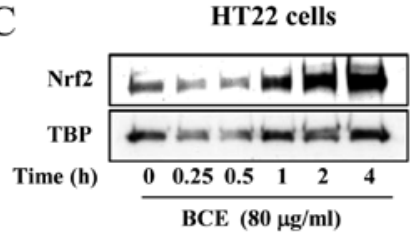

D
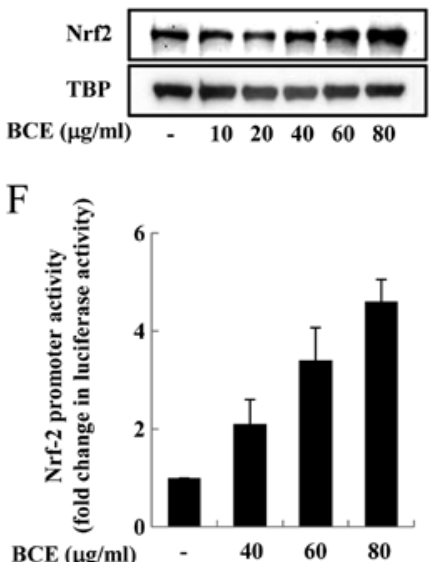

Figure 6. BCE induces the nuclear translocation and transactivation function of Nrf-2 in microglial BV2 and hippocampal HT22 cells. The nuclear protein extracts from both cell types were analyzed by western blotting. (A) Microglial BV2 and (C) hippocampal HT22 cells were treated with $80 \mu \mathrm{g} / \mathrm{ml}$ of BCE for 0.25-4 h. (B and D) Both cell types were treated with 10, 20, 40, 60 and $80 \mu \mathrm{g} / \mathrm{ml}$ of BCE for 0.5 and $4 \mathrm{~h}$, respectively. (E and F) Microglial BV2 and hippocampal HT22 cells were treated with different amounts of BCE $(0,40,60,80 \mu \mathrm{g} / \mathrm{ml})$, transfected with a Nrf-2-responsive ARE-luciferase construct and then harvested after $24 \mathrm{~h}$. Relative luciferase counts were compared with those in the untreated control cells. 


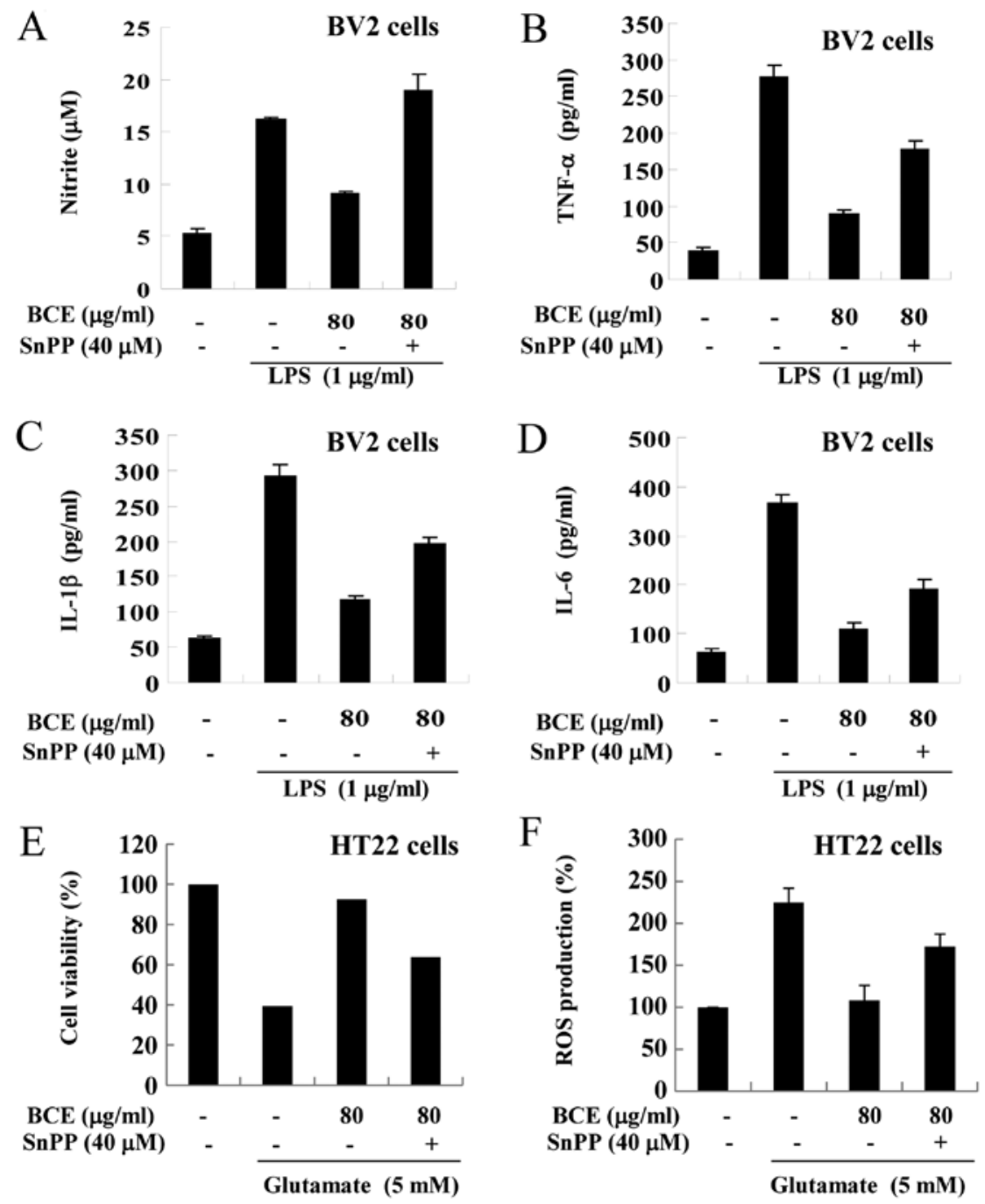

Figure 7. Effects of BCE on the production of pro-inflammatory cytokines in microglial BV2 cells and on glutamate-induced neurotoxicity in hippocampal HT2 2 cells. Microglia BV2 cells were treated with $80 \mu \mathrm{g} / \mathrm{ml} \mathrm{BCE}, 1 \mathrm{~h}$ prior to incubation with SnPP (40 $\mu \mathrm{M})$ and/or LPS (1 $\mu \mathrm{g} / \mathrm{ml})$. (A) Nitrate production was determined using the Griess reaction. (B) TNF- $\alpha$, (C) IL-1 $\beta$ and (D) IL-6 present in the culture supernatants were measured by ELISA assay. (E and F) After treatment with $80 \mu \mathrm{g} / \mathrm{ml}$ of BCE, the hippocampal HT22 cells were exposed to SnPP (40 $\mu \mathrm{M})$ and/or glutamate (5 mM). (E) Cell viability was measured by the MTT assay. (F) ROS production was determined using quantitative flow cytometry.

Nuclear accumulation of Nrf-2 increased with increasing doses of BCE, especially in the cells treated with BCE for $4 \mathrm{~h}$ (Fig. 6C). The nuclear accumulation of Nrf-2 increased with increasing doses of $\mathrm{BCE}$, in a concentration-dependent manner (Fig. 6D). To examine whether BCE also induces Nrf-2-mediated transactivation of target genes, we performed reporter gene analysis using an ARE-containing luciferase reporter construct. Microglial BV2 and hippocampal HT22 cells were transfected with the Nrf-2-responsive luciferase vector and then treated with $0,40,60$, or $80 \mu \mathrm{g} / \mathrm{ml}$ of BCE. We observed that the luciferase activity increased with increasing doses of BCE, indicating an increase in Nrf-2-responsive promoter activity (Fig. 6E and F). This data indicated that BCE induces Nrf-2 translocation from the cytoplasm into the nucleus and that Nrf-2 binds the ARE in the promoter region. This Nrf-2-mediated transactivation is involved in the expression of a number of genes, including HO-1, involved in anti-inflammatory and antioxidant pathways. Therefore, we hypothesized that BCE induces the expression of HO-1 through the Nrf-2-ARE pathway.
Confirmation of the anti-inflammatory and antioxidant effects induced by BCE. In order to confirm that the antiinflammatory and antioxidant effects of BCE occurred through the HO-1 signaling pathway, we conducted experiments using a selective inhibitor of HO-1 (SnPP). First, we examined the expression levels of pro-inflammatory cytokines in microglial BV2 cells. After treatment with $80 \mu \mathrm{g} / \mathrm{ml}$ of BCE, microglial BV2 cells were exposed to SnPP $(40 \mu \mathrm{M})$ and/or LPS $(1 \mu \mathrm{g} / \mathrm{ml})$ (Fig. 7A-D). The production of NO increased in only the LPS-stimulated cells and decreased in cells pre-treated with BCE $(80 \mu \mathrm{g} / \mathrm{ml})$ (Fig. 7A). These effects were reversed in SnPP-treated cells. A similar reversal was observed in the production of TNF- $\alpha$, IL-1 $\beta$ and IL-6 (Fig. 7B-D). Next, we examined whether the cytoprotective effects of BCE were HO-1-dependent. After treatment with $80 \mu \mathrm{g} / \mathrm{ml}$ of BCE, hippocampal HT22 cells were exposed to SnPP $(40 \mu \mathrm{M})$ and/or glutamate $(5 \mathrm{mM})$. The viability of cells was reduced in cells treated with glutamate alone, and the glutamate-induced decrease in cell viability was rescued with BCE treatment; however, this rescue was absent in 
SnPP-treated cells (Fig. 7E). Taken together, these results demonstrate that the mechanism behind $\mathrm{BCE}$ cytoprotective and $\mathrm{ROS}$ reduction activity is dependent on $\mathrm{HO}-1$ activity.

\section{Discussion}

Recent studies suggest that neuroinflammation contributes to the development of neurodegenerative diseases $(11,12)$. Furthermore, several studies have shown that anti-inflammatory reactions play an important role in reducing cytotoxicity $(24,25)$. The results of our study showed that BCE may protect hippocampal HT22 and microglial BV2 cells from glutamateand LPS-induced cytotoxicity, respectively. To investigate whether BCE has neuroprotective and anti-neuroinflammatory effects, we conducted two types of experiments. We determined whether BCE had antioxidant effects (i.e., removal of ROS), and in the second experiment we determined whether BCE had anti-inflammatory effects (i.e., decrease of proinflammatory cytokines, including TNF- $\alpha$, IL- $1 \beta$ and IL-6). We discovered that BCE exerted both an antioxidant and an anti-inflammatory effect in neuronal cells.

LPS is an endotoxin and is found in gram-negative bacteria, as a constituent of the outer membrane. It induces innate immunity, including production of pro-inflammatory mediators such as NO, TNF- $\alpha$ and IL-6 (26). We discovered that BCE significantly decreased the production of pro-inflammatory mediators and cytokines in LPS-stimulated microglial BV2 cells (Fig. 2). Notably, a previous study revealed that BC extract had anti-inflammatory effects in a murine model of asthma (27). These data suggested that BC has strong antiinflammatory properties in neurons and in airway cells.

ROS are essential molecules that regulate the redox status and growth of cells (28). ROS are highly reactive as they have at least one unpaired electron. Therefore, excessive production of ROS may promote harmful reactions that may cause both reversible and irreversible tissue damage. ROS are implicated in a number of disorders including neurological diseases, cancer, Parkinson's and Alzheimer's disease (29). The level of ROS is important since excessive amount of ROS cause oxidative stress and cell damage by inducing harmful reactions (28). Therefore, the ROS-scavenging activity of candidate substances is a suitable barometer of the potential antioxidant effects. To validate the antioxidant effects of BCE in microglial BV2 and hippocampal HT22 cells, we examined whether BCE reduces the production of ROS using flow cytometry. Our data showed that BCE provides a cytoprotective effect through the reduction of ROS levels in both hippocampal HT22 and microglial BV2 cells (Fig. 4).

A previous study reported that the upregulation of HO-1 protects cells by degrading pro-oxidant heme to oxidantscavenging bile pigments, including BV and BR. Moreover, it focused on the relationship between the neuroprotective effects and HO-1 signaling (12). In this study, we confirmed that BCE induces HO-1 expression through the Nrf-2 signaling pathway (Figs. 5 and 6). We discovered that BCE induces the upregulation of $\mathrm{HO}-1$, both at the protein and mRNA levels using western blotting and qRT-PCR (Fig. 5). Further, to identify whether BCE induces Nrf-2 translocation from the cytosol to the nucleus, we examined the Nrf-2 level in the nuclear fraction and the promoter activity by using
ARE-reporter plasmid. Results showed that BCE significantly induced the expression of $\mathrm{HO}-1$ and translocation of Nrf-2 (Fig. 6).

The data presented in Fig. 7 clearly demonstrate a decisive role for HO-1 enzymatic activity in the neuroprotective and anti-neuroinflammatory effects exerted by BCE. We used an HO-1 inhibitor (SnPP) to confirm that the protective effects of $\mathrm{BCE}$ were due to HO-1 signals. The anti-neuroinflammatory effect of BCE was reversed by SnPP in microglial BV2 cells (Fig. 7A-D). In addition, the protective effects of BCE on cell viability and ROS production was also reversed by SnPP in hippocampal HT22 cells (Fig. 7E and F). Thus, it is possible that neuroprotective and anti-neuroinflammatory effects of BCE may occur through HO-1 signaling pathways.

In conclusion, the findings of this study suggest that BCE exhibits neuroprotective and anti-neuroinflammatory effects in murine microglial BV2 and hippocampal HT22 cells. Furthermore, we determined that these effects occur through the Nrf-2/HO-1-mediated pathway. Our results provide a new insight for understanding the neuroprotective mechanisms of BCE. These effects of BCE, coupled with its low toxicity, point to a strong potential for use in the treatment of patients suffering from neurodegenerative diseases such as Alzheimer's disease.

\section{Acknowledgements}

This research was supported by the Basic Science Research Program through the National Research Foundation of Korea (NRF) funded by the Ministry of Education, Science and Technology (2012R1A12005031).

\section{References}

1. Selkoe DJ: The molecular pathology of Alzheimer's disease. Neuron 6: 487-498, 1991.

2. Vina J, Lloret A, Giraldo E, Badia MC and Alonso MD: Antioxidant pathways in Alzheimer's disease: possibilities of intervention. Curr Pharm Des 17: 3861-3864, 2011.

3. Dheen ST, Kaur C and Ling EA: Microglial activation and its implications in the brain diseases. Curr Med Chem 14: 1189-1197, 2007.

4. Perry VH: The influence of systemic inflammation on inflammation in the brain: implications for chronic neurodegenerative disease. Brain Behav Immun 18: 407-413, 2004.

5. Jeong GS, Lee DS, Kwon TO, Lee HS, An RB and Kim YC: Cytoprotective constituents of the heartwood of Caesalpinia sappan on glutamate-induced oxidative damage in HT22 cells. Biol Pharm Bull 32: 945-949, 2009.

6. Murphy TH, Miyamoto M, Sastre A, Schnaar RL and Coyle JT: Glutamate toxicity in a neuronal cell line involves inhibition of cystine transport leading to oxidative stress. Neuron 2: 1547-1558, 1989.

7. Nguyen T, Nioi P and Pickett CB: The Nrf2-antioxidant response element signaling pathway and its activation by oxidative stress. J Biol Chem 284: 13291-13295, 2009.

8. Jiang T, Huang Z, Chan JY and Zhang DD: Nrf2 protects against As(III)-induced damage in mouse liver and bladder. Toxicol Appl Pharmacol 240: 8-14, 2009.

9. Li W and Kong AN: Molecular mechanisms of Nrf2-mediated antioxidant response. Mol Carcinog 48: 91-104, 2009.

10. Maines MD: The heme oxygenase system: a regulator of second messenger gases. Annu Rev Pharmacol Toxicol 37: 517-554, 1997.

11. Schipper HM: Heme oxygenase-1 in Alzheimer disease: a tribute to Moussa Youdim. J Neural Transm 118: 381-387, 2011.

12. Schipper HM, Song W, Zukor H, Hascalovici JR and Zeligman D: Heme oxygenase-1 and neurodegeneration: expanding frontiers of engagement. J Neurochem 110: 469-485, 2009. 
13. Schipper HM: Heme oxygenase expression in human central nervous system disorders. Free Radic Biol Med 37: 1995-2011, 2004.

14. Shibata M, Yamatake Y, Sakamoto M, Kanamori M and Takagi K: Phamacological studies on bamboo grass (1). Acute toxicity and anti-inflammatory and antiulcerogenic activities of watersoluble fraction (Folin) extracted from Sasa albomarginata Makino et Shibata. Nihon Yakurigaku Zasshi 71: 481-490, 1975 (In Japanese).

15. Brown GC and Neher JJ: Inflammatory neurodegeneration and mechanisms of microglial killing of neurons. Mol Neurobiol 41: 242-247, 2010.

16. Shin DH, Shin JY, Kim SH, Kim JH, Chung HJ and Kim JC: Single oral dose toxicity study of Bambusae caulis in Taeniam in rats. J Toxicol and Public Health 20: 325-328, 2004 (In Korean).

17. Nakamura Y, Si QS and Kataoka K: Lipopolysaccharideinduced microglial activation in culture: temporal profiles of morphological change and release of cytokines and nitric oxide. Neurosci Res 35: 95-100, 1999.

18. Liu J, Li L and Suo WZ: HT22 hippocampal neuronal cell line possesses functional cholinergic properties. Life Sci 84: 267-271, 2009.

19. Meda L, Cassatella MA, Szendrei GI, Otvos L Jr, Baron P, Villalba M, Ferrari D and Rossi F: Activation of microglial cells by beta-amyloid protein and interferon-gamma. Nature 374: 647-650, 1995

20. Fang J, Qin H, Seki T, Nakamura H, Tsukigawa K, Shin T and Maeda $\mathrm{H}$ : Therapeutic potential of pegylated hemin for reactive oxygen species-related diseases via induction of heme oxygenase-1: results from a rat hepatic ischemia/reperfusion injury model. J Pharmacol Exp Ther 339: 779-789, 2011.
21. Jung KA and Kwak MK: The Nrf2 system as a potential target for the development of indirect antioxidants. Molecules 15: 7266-7291, 2010.

22. Dennery PA: Regulation and role of heme oxygenase in oxidative injury. Curr Top Cell Regul 36: 181-199, 2000.

23. Surh YJ, Kundu JK, Li MH, Na HK and Cha YN: Role of Nrf2mediated heme oxygenase-1 upregulation in adaptive survival response to nitrosative stress. Arch Pharm Res 32: 1163-1176, 2009.

24. Ben-Hur T, Ben-Menachem O, Furer V, Einstein O, Mizrachi-Kol R and Grigoriadis N: Effects of proinflammatory cytokines on the growth, fate, and motility of multipotential neural precursor cells. Mol Cell Neurosci 24: 623-631, 2003.

25. Ekdahl CT, Claasen JH, Bonde S, Kokaia Z and Lindvall O: Inflammation is detrimental for neurogenesis in adult brain. Proc Natl Acad Sci USA 100: 13632-13637, 2003.

26. Seo T, Cha S, Kim TI, Lee JS and Woo KM: Porphyromonas gingivalis-derived lipopolysaccharide-mediated activation of MAPK signaling regulates inflammatory response and differentiation in human periodontal ligament fibroblasts. J Microbiol 50: 311-319, 2012.

27. Ra J, Lee S, Kim HJ, Jang YP, Ahn H and Kim J: Bambusae Caulis in Taeniam extract reduces ovalbumin-induced airway inflammation and T helper 2 responses in mice. J Ethnopharmacol 128: 241-247, 2010

28. Davies KJ: Oxidative stress: the paradox of aerobic life. Biochem Soc Symp 61: 1-31, 1995.

29. Maeda $\mathrm{H}$ and Akaike T: Oxygen free radicals as pathogenic molecules in viral diseases. Proc Soc Exp Biol Med 198: 721-727, 1991. 